\title{
Nest architecture is linked with ecological success in songbirds
}

\author{
Iliana Medina ${ }^{1}$, Daniela Perez ${ }^{2}$, Ana $_{\text {Silva }}^{3}$, Justin Cally ${ }^{1}$, Constanza Leon ${ }^{4}$, Odile Maliet ${ }^{5}$, \\ and Ignacio Quintero 5 \\ ${ }^{1}$ University of Melbourne School of BioSciences \\ ${ }^{2}$ Universidade Federal do Parana \\ ${ }^{3}$ Universite de Lille \\ ${ }^{4}$ Australian National University \\ ${ }^{5}$ Institut de Biologie de l'Ecole Normale Superieure
}

July 21, 2021

\begin{abstract}
Bird nests are essential structures that directly determine the fitness of an organism. While there is theory and evidence predicting an association between species nest traits and their habitat, few studies have comprehensively examined the macroevolutionary patterns driving nest evolution, species niche and their interrelation. Using information on 3174 species of songbirds, we show that species that build domed nests (i.e. nests with a roof) have smaller ranges, narrower thermal niches, are less likely to colonise urban environments and have potentially higher extinction rates compared to species that build open nests. Moreover, we show that these macroevolutionary patterns could be driven by the higher energetic demands when building domed nests, which consumes more time and might restrict breeding opportunities. These diverse strands of evidence suggest that the transition from domed to open nests in passerines represents an important evolutionary innovation behind the success of the largest radiation of birds.
\end{abstract}

\section{Introduction}

Nests are fundamental structures for avian reproduction that determine egg survival and development (Deeming et al. 1991; Deeming \& Mainwaring 2015). The thermal environment inside these natural incubators influences an individual's fitness, and therefore, nest traits that affect internal conditions are expected to be under strong selective pressures (Heenan 2013; Mainwaring et al. 2014). Songbirds (suborder Passeri) and their more than 4500 species represent the largest extant bird radiation and are known to build a variety of nest types. The majority of species in this clade build 'open nests', that is, cups or platforms where nest contents are exposed. In contrast, some species, like Australian lyrebirds, African weavers and penduline tits, build domed nests, which are structures characterised by an enclosing roof and a side entrance. Domed nests have often been suggested to reduce predation and offer thermal insulation, protecting nest contents better than open nests (Collias 1997; Lamprecht \& Schmolz 2004; Martin et al. 2017). Recent evidence highlights their thermoregulatory advantages in colder environments by enabling parents to spend more time away from their nests (Lamprecht \& Schmolz 2004; Martin et al. 2017; Matysioková \& Remeš 2018), and in extremely hot environments by shielding nests from solar radiation (Griffithet al. 2016). Given the presumed advantages of domed nests over open nests, it remains a mystery why most birds in the world ( $70 \%$ of all avian families, $72 \%$ of songbird species) build open nests.

For years it was thought that selective pressures, imposed by temperature or predation rates, would have favoured the transition from open to domed nests in some clades (Collias 1997; Hansell 2000; Price \& Griffith 2017). The evolutionary history of nests in passerines, however, suggests the opposite: the common trait of building an open nest is a derived condition, an innovation. In 2017, Price and Griffith found evidence that 
the ancestral type of nest in passerines is domed, and this result is further supported by other comparative analyses (Fanget al. 2018; Medina 2019). Thus, rather than selection favouring the occurrence of domed nests throughout the evolution of passerines, this trait has been repeatedly lost. Furthermore, the transition from domed to open nests in passerines has been hypothesized to be a key innovation, given the subsequent diversification, colonization and expansion of open nest building lineages (Price \& Griffith 2017; Fanget al. 2018). We currently ignore why domed nests transitioned into open structures, but it is possible that costs associated with nest building could have led to the repeated loss of domed nests. For instance, while we lack information on whether building domed nests represents a higher energetic cost over building open nests, nest building is considered a costly activity and domed nests are heavier relative to the size of the builder when compared to open nests (Hansell 2000; Mainwaring \& Hartley 2013). Yet, we still lack formal analyses at large taxonomic scales to understand the underlying evolutionary processes and life-history implications of the transition between domed and open nests.

The type of nest a species builds could be tightly linked with its ability to live across different habitats or environments. For instance, if domed nests offer greater protection across a wider range of environments, then we would expect species with domed nests to sustain broader climatic tolerances, thereby enabling them to inhabit a wider range of habitats. On the other hand, if domed nests are a costly ancestral adaptation to now scarce specific habitats, then domed-nest lineages should exhibit narrower and more specialized environmental tolerances than their open-nest counterparts. An increased availability of milder habitats (in terms of climate and/or predation) could have released lineages from the costs of domed nest-building, resulting in larger populations and larger ranges, and potentially, an increase in speciation or reduction in extinction rates (Rosenzweig 1995). Indeed, Australian species with open nests tend to have larger ranges, wider climatic niches and are less likely to be under an IUCN threat category compared with species that build domed nests (Medina 2019).

Our main aim is to understand the evolutionary success of open nests by studying the potential ecological and evolutionary trade-offs associated with different nest types. First, we compare the building costs between domed and open nests using published information on time spent constructing the nest. We then test whether the evolution of domed and open nests in passerines is associated with differences in range size, climatic niche breadth, colonisation of novel environments and differences in speciation or extinction rates. We use a comprehensive macroevolutionary framework to explore for the first time the joint evolutionary dynamics of a critical component of the extended phenotype of birds.

\section{Methods}

\section{Nest type and other species traits}

We extracted species-level nest information from the website Birds of the World (BW) (Billerman et al. 2020) and Beruldsen (1980). We searched for information on all species of songbirds (suborder Passeri), following the taxonomy from Jetz et al. phylogenies (4685 species), with manual resolution of taxonomic names between BW and Jetz et al. For each species we collected information on nest type, which was classified into two broad categories: domed (defined as having a side entrance or a roof, or as being globular) or open (defined as a cup or a platform, or cases where there is only a mat of leaves, or vegetable material). Additionally, we recorded whether the species was a cavity nester or not, whether the species built both domed and open nests, or whether the nest was described as a pouch. This information was collected because in some species (e.g. Icteridae) nests are so elongated that they effectively look more liked domed than open nests. With these variables we generated fine scale classifications (six categories: open, domed, open nest inside cavity, domed nest in cavity, both types and pouch), and this classification was used in initial analyses. Based on results, we further merged data into broader categories, to increase sample size in each category. The main nest categories used were: domed, open or cavity nesters, or just domed vs. open nests (further explained in statistical analyses). Our final dataset included 3175 species with nest information (68\% of all Passeri).

Given that response variables like range size can be associated with body size and species latitude, we also 
collected information on body size for each of the species from Wilman et al. (2014) and information on the mean latitude from their distribution range from Sheard et al. (2020), to control for the effects of these variables. As a measure of colonisation of novel habitats we also collated information of species' presence in urban environments (data from IUCN, collated in Ducatez et al. (2020). This was recorded as 1 (presence) or 0 (no presence).

To test whether there are differences in the costs of nest building across nest types, we used the time spent building nests as proxy of the energetic cost. We searched for information on the average number of days that takes a species to construct a nest, if a range was given, we used the middle of the range as representative for the species. We also collected information on who builds the nest (females, males or both), given this could affect the time of nest construction.

\section{Environmental variables}

To test whether there are differences in niche width and range size between species with different nest types we used geographical distribution information from BirdLife (2019). We used a published dataset (Cally et al. 2021) of 19 bioclimatic variables from Worldclim (Fick \& Hijmans 2017) (details in Cally et al. 2021). These variables were sampled in 1000 random points across the distribution of each species, and provide information on temperature and rainfall across the range. We also extracted information on range size from Cally et al. $2021(n=3174)$. For a smaller set of species for which breeding range information was available $(\mathrm{n}=3049)$, we extracted information on the same 19 bioclimatic variables following the protocol in Cally et al. 2021, but this time we used a more recently developed dataset (CHELSA), which has an algorithm that predicts precipitation patterns more precisely than Worldclim (Karger \& Zimmermann 2019). For each climatic variable, and each species, we calculated the standard deviation across the 1000 points sampled, to estimate climatic variability across the species range (and breeding range). Since species restricted to islands are limited both in the extent of their range and the niche width, we performed analyses using both the whole dataset and only continental species.

To summarise information on variation in temperature and variation in precipitation across a species range, we performed two principal component analyses, one for temperature and one for precipitation variables. We split precipitation from temperature because we expected that nest types would be more linked to temperature than precipitation variables, given the proposed thermoregulatory capabilities of domed nests (Martin et al. 2017). The same was done for the breeding range, leading to four principal component analyses that summarise information on how variable temperature and precipitation are both within total and only breeding ranges. We report the PC loadings and percentage of variance explained for PCs. We refer to the first principal component from temperature variables as $\mathrm{PC}_{\mathrm{TEMP}}$ and to the first principal component based on precipitation variables as $\mathrm{PC}_{\mathrm{PRE}}$. These $\mathrm{PCs}$ (four in total) were used each as a different response variable in statistical analyses, but many of them were very highly correlated (e.g. $\mathrm{r}^{2}=0.99$, supp. material Figure 1). Given that results were very similar for both breeding and whole range, we present in the main text the results from the whole range, because it holds a larger sample.

\section{Tip-level regression analyses}

Information on days taken to build nest was available for 277 species (69 domed, 208 open), and to test whether there are differences in the time taken to build a nest we used linear models that account for evolutionary history (PGLS, description of approach below). We used the time taken to build in days as response variable (log transformed) and we used log body size, the sex of the builder (female, both or male) and the type of nest built (domed/open) as predictors.

To test whether building different nest types is associated with current range size and niche width we used linear models that account for evolutionary history, with climatic niche width $\left(\mathrm{PC}_{\mathrm{TEMP}}\right.$ or $\left.\mathrm{PC}_{\mathrm{PRE}}\right)$, range size or presence in urban environments (yes/no) as response variables. As predictors, we used the species nest type and body size $(\log )$, because body size is known to explain variation in range size in birds (Gaston \& Blackburn 1996). In the case of range size we also accounted for the absolute mid-latitude of the species range, since tropical species are expected to have smaller ranges (Gaston et al. 1998). For initial analyses 
we used as 'nest type' a detailed classification of nest type with six categories (domed, open, domed in cavity, open in cavity, pouch and both). Based on non-significant differences across some categories, and for simplicity, for posterior analyses we used a more intuitive classification (domed, open and cavity). In this case, we consider cavity nesting species those that build either domes or cups inside cavities or crevices. We also consider species building pouches or both domed and open nests to have 'open' nests, because pouch nests do not have a roof and open nests seems to be the derived state. For diversification analyses we also created a simpler category based exclusively on the structure built, splitting species only between domed and open nests, since building inside cavities (domes or cups) represents the nesting site preference rather than nest structure per se. Analyses were done using multiple categorisations, to ensure results were consistent independently of how we categorised nest type. We used the R package 'performance' and the command check_model to detect outliers, and assess whether there were any collinearity issues in our set of predictors (Lüdecke et al. 2019).

For the continuous response variables (time spent building nest, $\mathrm{PC}_{\mathrm{TEMP}}, \mathrm{PC}_{\mathrm{PRE}}$ and range size) we used a phylogenetic generalised least squares regression (PGLS), using maximum likelihood to estimate lambda, implemented in the R package 'caper' (Orme 2013). To control for phylogenetic relatedness among species we a generated a maximum clade credibility tree (MCC, across 10000 trees) using the package 'Phangorn' (Schliep 2011) and a set of 10000 phylogenies from birdtree.org (Jetz et al. 2012). For models with significant results using the MCC tree, we also performed PGLS analyses across a set of 100 trees. For each model using the MCC tree we report the estimates and p-values calculated, and for the analysis on 100 trees we generated highest posterior density intervals (HPD) for the estimates using the R package 'coda' (Plummer et al. 2006). We highlight that these tip-level regressions inform us on the links between multiple variables and independent origins of such associations, but they do not inform us about the processes underlying such associations (see below for such analyses).

For the binary response variable (urban or not) we used a Bayesian approach in the R package MCMCglmm (Hadfield 2010). Predictors were nest type (open, domed, cavity) and log body size. This nest category was used based on the results of the analysis described in the previous paragraph. We run one model using the MCC tree as a random effect until convergence was reached. To account for phylogenetic uncertainty, we followed Ross et al. (2013). Briefly, we run the model using 1300 different trees and for each tree used 10000 iterations and saved the last iteration before going into the next tree. We used the first 300 iterations (e.g. 300 trees) as burnin and assessed model convergence, ensuring that the effective sample size was above 900. We report the credibility intervals for each predictor in each model.

\section{Diversification analyses and evolutionary models}

We estimated speciation rates across a range of phylogenetic trees using the

cladogenetic diversification rate shift (ClaDS) Bayesian model (Malietet al. 2019) that infers branch-specific diversification rates. ClaDS allows for gradual variation in diversification rates and was shown to perform well in identifying both small and large rate shifts (Maliet et al. 2019). We used the model that accounts for a scenario with a constant turnover rate (ClaDS2 model) and the faster implementation in Julia (v.1.1.0, (Bezanson et al. 2017) that uses data augmentation (Maliet \& Morlon 2020), setting the sampling probability to 0.63 . To test whether nest type is associated with variation in speciation rates, we used the tip-rate estimates (estimates for each terminal branch at present) extracted from ClaDS as response variable in a PGLS model, with nest type and $\log$ (range size) as predictors, since range size has been shown to be associated with speciation (Cally et al. 2021). The nest type category used for this model was open vs. domed, given that we are interested in the actual nest building behaviour, not where the nest is placed. A main model was run using the MCC tree and we also performed the same analysis in a sample of 20 phylogenetic trees to account for phylogenetic uncertainty.

To gain a more mechanistic understanding on the feedback between species traits and their influence on diversification, we used the Multiple State Speciation Extinction (MuSSE) model to jointly estimate state dependent speciation and extinction rates (FitzJohn 2012). Modelling trait evolution while accounting for the 
diversification process results in more appropriate estimates of trait transition rates and ancestral estimates, while accounting for uncertainty in the species for which we do not have state information (Maddison et al. 2007; FitzJohn 2012). We modelled the correlated evolution of niche width andnest type data by aggregating them into four different states: narrow niche and domed nest, narrow niche and open nest, wide niche and domed nest and wide niche and open nest. We assigned a species as having a wide or narrow niche based on whether the $\mathrm{PC}_{\text {TEMP }}$ was below or above the median value of the whole dataset $\left(\mathrm{PC}_{\text {TEMP }}=-0.639\right)$. Because we are interested in the interaction among these states, we disallowed transition rates between states that effectively would represent simultaneous changes in nest type and niche width (e.g. going from wide niche and domed nest directly to narrow niche and open nest is not allowed).

Additionally, to allow for unobserved taxonomic variance in the diversification process, thereby not forcing the model to explain all the heterogeneity in diversification, we ran HiSSE (Hidden State Speciation Extinction) with two concealed states (Beaulieu \& O'Meara 2016). Both analyses were performed using the MCC tree within a Bayesian Framework using RevBayes, including ancestral state estimation (details in supplementary material). To evaluate the effect of phylogenetic uncertainty, we ran the Maximum Likelihood approach to MuSSE implemented in the R package 'castor' (Louca \& Doebeli 2018) across 20 phylogenetic trees. Because phylogenetic uncertainty did not influence the results, and results were consistent across methods, we present the HiSSE analyses in the main text and castor and MuSSE results in the supplementary material. Finally, given that the uncertainty of species position in the phylogeny might be higher for some states, potentially biasing transition rates, we implemented an additional analysis that specifically accounted for missing data per character state (details in supplementary material).

\section{Results}

From all species considered in this study ( $\mathrm{n}=3174$, Figure 1), 60\% (1902 spp.) were classified as having an open nest, $25 \%$ (790 spp.) were classified as domed nesters and 15\% (482 spp.) were classified as cavity nesters. Only $1.8 \%$ of the species (60 spp.) build both types of nests. From the cavity nesters $17 \%$ build domed nests inside the cavities and $83 \%$ build cups. Most species were classified as continental (2596 spp.) and 578 spp. were classified as insular.

Species that build domed nests spend more time building their nests than species with open nests $(\beta=-0.37$ [HPD 95\%: $-0.40,-0.36]$, t-value $=-3.31, \mathrm{p}=0.001$, Figure 2$)$. Larger species take longer to build nests $(\beta=$ $0.14[0.11,0.14], \mathrm{t}$-value $=2.61, \mathrm{p}=0.009)$ and couples have a weak tendency to take longer to build nests $(\beta$ $=0.13[0.15,0.19]$, t-value $=1.923, \mathrm{p}=0.055)$.

Body size, latitude and nest type were correlated (Figure S2), but there was enough variation across species to avoid multicollinearity issues, and all variance inflation factors were low (max 2.18), and well below the recommended threshold of five. Species that build domed nests have smaller geographic ranges, after controlling for the effect of latitude and body size (Figure 3, Figure S2). They also have narrower temperature niches $\left(\mathrm{PC}_{\mathrm{TEMP}}\right)$, but not precipitation niches $\left(\mathrm{PC}_{\mathrm{PRE}}\right)$, when compared with species with open nests (Table 1, Figure 3, Figure S3). This pattern was similar when comparing domed and open nests built within cavities (Figure S3). There were no differences between open and cavity nesters in range size or precipitation niche width, but there were differences in $\mathrm{PC}_{\text {TEMP }}$ (Table 1), with cavity nesters having broader thermal niches. Similar trends were found when using either continental or all species, when using whole range or breeding range information and when using alternative classifications of nest type (Tables S2 and S3, Figure S4). Species that live in urban environments are usually species with open and not domed nests $(\beta=-1.858, \mathrm{p}=$ 0.001, Table S4, Figure S5), but there were no differences between open and cavity nesters (Table S4).

There were no differences in speciation rates between species with different nest types when using tip-rates derived from the ClaDS model. Although when using the MCC tree we found significantly higher rates for open nests (Domed vs. Open, $\beta=0.051$, t-value $=2.36, p=0.017$ ), when we sampled 20 random trees there was no association (HPD interval $\beta=-0.065$ to 0.030 , p-value $=0.03$ to 0.83 ). The MuSSE analysis showed differences in both speciation and extinction rates between the four categories (Table S5, Figure $4 \mathrm{~A}$ ), where species with domed nests had higher average extinction rates (in 18 out of 20 trees) and species 
with narrow niches had higher speciation rates (in 12 out of 20 trees). The HiSSE analysis showed similar patterns (Figure 4A, Table S5). A similar pattern was detected in the analyses that employed only species with genetic data (for extinction at least), but as expected, this pattern was much weaker than when using the full dataset.

The most likely ancestral state according to all analyses for the clade Passeri was having a small niche and a domed nest, although as usual, reconstruction based only on extant species should be taken with precaution. The pattern reported was evident from the HiSSE analysis but was more ambiguous in the MuSSE analyses, where probabilities of state 1 (small niche and domed nest) and state 2 (large niche and domed nest) were very similar (Table S5). The transition rate matrix in all analyses (including the one using only species with genetic data), showed that transitions between niche widths (small/large) are more common than transitions between nest types (Figure 4B, Figure S6-S8), and that the most likely route to the association between open nests and wide niches (the most common and derived state) would have occurred from domed nest/narrow niche, to open nest/narrow niche, and then open nest/ wide niche (Figure 4B). This path is over 11 times more likely than evolving a wider niche and then transitioning to an open nest, and suggests that a transition in nest type, from domed to open, is the first step to then support a wider niche.

\section{Discussion}

Nest construction is suggested to be an important innovation that facilitated the radiation of modern birds, specially passerines (Collias 1997; Collias \& Collias 2014). Our results support the idea that nests could be important in the ecological success of birds and further suggest that the transition from domed to open nests in songbirds could have contributed to the expansion and success of this radiation in the world. We found that species with open nests spend less time building their nests when compared to species with domed nests. Species with open nests also have larger ranges, broader thermal niches, are more likely to colonise urban environments and have lower extinction rates than species with domed nests.

Collias (1964 \& 1997) suggested several benefits of building open nests. First, they are often made of fewer materials. Second, because they are smaller, they can be hidden from predators and can also allow a quicker escape from predator attack. Third, they should take less time and energy to build, which would make renesting during the breeding season more likely. Our results support the hypothesis that open nests reduce energy and time expenditure; we found that species building open nests take significantly less days to build, especially when the nest is built by females or a couple. This pattern could be explained by the large size of domed nests, since open cups have a lower weight compared to domed nests, relative to the size of the builder (Hansell 2000). Evolutionary transitions from domed to open nests, hence, could be favoured given the higher costs associated with building domed nests.

Our regression results also show that species with open nests have wider ranges and broader climatic niches compared to species with domed nests, a pattern previously reported for Australian passerines (Medina 2019). These findings support the idea that open nests have been important in the radiation of passerines, as explicitly suggested by Price and Griffith (2017); Fang et al. (2018). A transition to less costly open nests could have allowed further expansion of a clade into novel environments, and their evolutionary success. This interpretation should be treated with caution, though, since the link between range size (or climatic niche) and nest type that we report in the tip-level analyses is not evidence of causation. Another explanation for such link is that the change in nest type occurred after a transition in niche width. As species colonised new environments that are less thermally demanding, selection could have favoured another type of nest (i.e. an open nest). However, our results from the MuSSE and HiSSE models do not support this latter hypothesis, since transition rates from all analyses suggest that changes in niche width were preceded by changes in nest type. The likely route to the most common combination of characters - broad niches and open nests is from ancestors with narrow niches and open nests, indicating first a change in nest type (to open) and a subsequent expansion in niche (and hence range). Thus, our findings suggest that building open nests could be important in the geographical expansion and the success of modern Passeri.

How could a transition to open nests be linked with range and niche expansion? Our study does not offer 
insights into the precise mechanism, but we offer a potential scenario. Differences in the time taken to build nests could affect the number of nests a species builds in a breeding season, leading to differences in fecundity and explaining the macroevolutionary patterns reported here. In North American and European birds there are differences in annual fecundity between species with different nest types, and species with open nests have a higher number of broods per year (Böhning-Gaese et al. 2000). Furthermore, fecundity can have important broad scale impacts. Laube et al.(2013) found a link between life history traits associated with fecundity (e.g. clutch size and clutches per year) and the range size of European passerines. Higher fecundity can lead to a higher local abundance, and ultimately allow range expansion (Blackburn et al.2006). A link between fecundity and range size (and niche breadth) has been shown in other clades as well (Jelbert et al. 2015; Janet al. 2019). If open nests lead to higher fecundity (because of more re-nesting opportunities), then this could result in larger ranges and broader niches in species with open nests. Testing for differences in fecundity between species with open and domed nests is beyond the scope of our study, but it would be interesting to test whether the costs of nest building could affect fecundity, and if this path could explain the broad scale patterns reported here.

Our analyses also suggest that clades with open nests present lower extinction rates compared to those that build domed nests, offering additional support to the idea of open nests as important in the passerine radiation. We found differences in extinction rates that are consistent across trees and linked specifically to nest type. Although it has been shown that species with broader ranges tend to have both lower extinction risk and also lower speciation rates (Purvis et al. 2000; Greenberg \& Mooers 2017), we found that open nesting species (with either small or wide niches/ranges) tend to have lower extinction rates compared to species with domed nests. We, however, strongly highlight that estimates of extinction rates from molecular phylogenies should be taken with extreme caution (Rabosky 2010) and differences in rates were smaller when the dataset used included only species with genetic data. In any case, our results do not support the hypothesis of higher speciation rates in species with open nests, and instead show that high speciation rates are mostly related to small range sizes, supporting previous findings (Cally et al. 2021). Therefore, the relative success of open nesting species cannot be attributed to higher speciation rates - supporting Price and Griffith (2017) - but could potentially be linked to a higher risk of extinction of dome nesting clades.

We also found evidence of higher urban presence for species with open nests, which could potentially be explained by links with range size, since species with open nests tend to have larger ranges, and urban species are also more likely to have large ranges (Reynolds et al. 2019). Our analysis showed, however, that even if range size is controlled for in the model, there is still an independent and significant effect of nest type on urban presence. To our knowledge, this is the first time a global link between nest architecture and urban life has been proposed. This link could be indirect and explained by selection on variables related to nest type. For instance, previous studies have shown that species living in cities have high rates of of annual fecundity (Møller 2009). If urban habitats constrain the presence of species with low fecundity, that could restrict dome nesting species. Also, non-urban species tend to nest on the ground (Sol et al.2014), and species with domed nests more commonly nest on the ground (Hall et al. 2015). Building nests on the ground might be challenging in urban environments. On the other hand, the link between urban environments and nest type could be direct: if open/cavity nests require less material or have higher flexibility in construction than domed nests, then there could be selection against dome nesters in cities. Urban environments could also be more thermally beneficial (e.g. more places with shade), in which case the thermal benefits of domed nests would not be required. In less harsh environments, species can adjust nest site selection to compensate for the lack of a nest roof (Slagsvold 1989; Kauffman et al. 2020). Future studies could investigate what is constraining the presence of dome nesting species in urban environments.

To conclude, our results show a macroevolutionary link between the type of nest that a bird builds and several traits associated with evolutionary success, such as range size, colonisation of urban environments and reduced extinction risk. Our analyses also suggest that transitions from domed to open nest type could have enabled such success. Combined, these findings support a scenario explicitly proposed by previous studies (e.g. (Price \& Griffith 2017; Fang et al.2018), where the evolution of open nests is a key innovation in passerines, sensu Stroud and Losos (2016); Rabosky (2017). Namely, although there is no evidence suggesting 
that species with open nests have higher speciation rates, having an open nest is linked with an increase in ecological opportunity (through an increase in range size), allowing the colonisation of novel habitats, including urban habitats, and potentially decreasing the risk of extinction. The precise microevolutionary mechanisms that have led to the associations reported in this study remain a mystery but a fruitful venue for targeted research exploring the costs and benefits of building different nest types.

\section{Acknowledgments}

We would like to thank all the researchers that collected the field data that was employed in the analyses presented in this manuscript. We would also like to express our gratitude to Sebastian Höhna for his help with the implementation of the RevBayes analyses. We also thank Luke Holman for assistance with the implementation of analyses in Spartan, Pat Backwell for supporting DP during the course of this research and the LIEF HPC-GPGPU facility hosted at the University of Melbourne.

\section{Funding}

This research was supported by ARC DECRA 200100500 to IM.

\section{Competing interests}

The authors declare no competing interests.

\section{References}

1.

Beaulieu, J.M. \& O'Meara, B.C. (2016). Detecting hidden diversification shifts in models of trait-dependent speciation and extinction.Systematic biology , 65, 583-601.

2 .

Beruldsen, G. (1980). A Field Guide to Nests 83 Eggs of Australian Birds . Rigby.

3.

Bezanson, J., Edelman, A., Karpinski, S. \& Shah, V.B. (2017). Julia: A fresh approach to numerical computing. SIAM review , 59, 65-98.

4 .

Billerman, S.M., Keeney, B.K., Rodewald, P.G. \& Schulenberd, T.S. (2020). Birds of the World. Cornell Laboratory of Ornithology, Ithaca, NY, USA, https://birdsoftheworld.org/bow/home.

5.

Blackburn, T.M., Cassey, P. \& Gaston, K.J. (2006). Variations on a theme: sources of heterogeneity in the form of the interspecific relationship between abundance and distribution. Journal of Animal Ecology , 75, 1426-1439.

6.

Böhning-Gaese, K., Halbe, B., Lemoine, N. \& Oberrath, R. (2000). Factors influencing the clutch size, number of broods and annual fecundity of North American and European land birds. Evolutionary Ecology Research , 2, 823-839.

7.

Cally, J.G., Stuart-Fox, D., Holman, L., Dale, J. \& Medina, I. (2021). Male-biased sexual selection, but not sexual dichromatism, predicts speciation in birds. Evolution.

8.

Collias, N.E. (1964). The evolution of nests and nest-building in birds.American Zoologist, 175-190. 
9.

Collias, N.E. (1997). On the origin and evolution of nest building by passerine birds. Condor , 99, 253-270.

10.

Collias, N.E. \& Collias, E.C. (2014). Nest building and bird behavior . Princeton University Press.

11.

Deeming, D. \& Mainwaring, M. (2015). Functional properties of nests.Nests, eggs and incubation: new ideas about avian reproduction, 29-49.

12.

Deeming, D.C., Deeming, D.C. \& Ferguson, M.W. (1991). Egg incubation: its effects on embryonic development in birds and reptiles. Cambridge University Press.

13.

Ducatez, S., Sol, D., Sayol, F. \& Lefebvre, L. (2020). Behavioural plasticity is associated with reduced extinction risk in birds. Nature Ecology $\&$ Evolution, 1-6.

14.

Fang, Y.-T., Tuanmu, M.-N. \& Hung, C.-M. (2018). Asynchronous evolution of interdependent nest characters across the avian phylogeny. Nature Communications , 9, 1863.

15.

Fick, S.E. \& Hijmans, R.J. (2017). Worldclim 2: New 1-km spatial resolution climate surfaces for global land areas. International Journal of Climatology , 37, 4302-4315.

16.

FitzJohn, R.G. (2012). Diversitree: comparative phylogenetic analyses of diversification in R. Methods in Ecology and Evolution, 3, 1084-1092.

17.

Gaston, K.J. \& Blackburn, T.M. (1996). Global scale macroecology: interactions between population size, geographic range size and body size in the Anseriformes. Journal of Animal Ecology, 701-714.

18.

Gaston, K.J., Blackburn, T.M. \& Spicer, J.I. (1998). Rapoport's rule: time for an epitaph? Trends in Ecology ES Evolution, 13, 70-74.

19.

Greenberg, D.A. \& Mooers, A.O. (2017). Linking speciation to extinction: Diversification raises contemporary extinction risk in amphibians. Evolution Letters , 1, 40-48.

20.

Griffith, S.C., Mainwaring, M.C., Sorato, E. \& Beckmann, C. (2016). High atmospheric temperatures and 'ambient incubation'drive embryonic development and lead to earlier hatching in a passerine bird.Royal Society Open Science, 3, 150371.

21.

Hadfield, J.D. (2010). MCMC methods for multi-response generalized linear mixed models: the MCMCglmm R package. Journal of Statistical Software , 33, 1-22. 
22.

Hall, Z.J., Street, S.E., Auty, S. \& Healy, S.D. (2015). The coevolution of building nests on the ground and domed nests in Timaliidae. The Auk, 132, 584-593.

23.

Hansell, M. (2000). Bird nests and construction behaviour . Cambridge University Press.

24.

Heenan, C.B. (2013). An overview of the factors influencing the morphology and thermal properties of avian nests. Avian Biology Research , 6, 104-118.

25.

Jan, P.-L., Lehnen, L., Besnard, A.-L., Kerth, G., Biedermann, M., Schorcht, W. et al. (2019). Range expansion is associated with increased survival and fecundity in a long-lived bat species. Proceedings of the Royal Society B , 286, 20190384.

26.

Jelbert, K., Stott, I., McDonald, R.A. \& Hodgson, D. (2015). Invasiveness of plants is predicted by size and fecundity in the native range. Ecology and Evolution, 5, 1933-1943.

27.

Jetz, W., Thomas, G., Joy, J., Hartmann, K. \& Mooers, A. (2012). The global diversity of birds in space and time. Nature, 491, 444.

28.

Karger, D.N. \& Zimmermann, N.E. (2019). Climatologies at High Resolution for the Earth Land Surface Areas CHELSA V1. 2: Technical Specification. Swiss Federal Research Institute WSL, Switzerland .

29.

Kauffman, K.L., Elmore, R.D., Davis, C.A., Fuhlendorf, S.D., Goodman, L.E., Hagen, C.A. et al. (2020). Role of the thermal environment in scaled quail (Callipepla squamata) nest site selection and survival.Journal of Thermal Biology, 102791.

30.

Lamprecht, I. \& Schmolz, E. (2004). Thermal investigations of some bird nests. Thermochimica Acta, 415, 141-148.

31.

Laube, I., Korntheuer, H., Schwager, M., Trautmann, S., Rahbek, C. \& Bohning-Gaese, K. (2013). Towards a more mechanistic understanding of traits and range sizes. Global Ecology and Biogeography, 22, 233-241.

32.

Louca, S. \& Doebeli, M. (2018). Efficient comparative phylogenetics on large trees. Bioinformatics , 34, 1053-1055.

33.

Ludecke, D., Makowski, D. \& Waggoner, P. (2019). Performance: assessment of regression models performance. $R$ package version $0.4,2$.

34. 
Maddison, W.P., Midford, P.E. \& Otto, S.P. (2007). Estimating a binary character's effect on speciation and extinction. Systematic biology, 56, 701-710.

35.

Mainwaring, M.C. \& Hartley, I.R. (2013). The energetic costs of nest building in birds. Avian Biology Research , 6, 12-17.

36.

Mainwaring, M.C., Hartley, I.R., Lambrechts, M.M. \& Deeming, D.C. (2014). The design and function of birds' nests. Ecology and Evolution, 4, 3909-3928.

37.

Maliet, O., Hartig, F. \& Morlon, H. (2019). A model with many small shifts for estimating species-specific diversification rates. Nature ecology \& evolution, 3, 1086-1092.

38.

Maliet, O. \& Morlon, H. (2020). Fast and accurate estimation of species-specific diversification rates using data augmentation.bioRxiv .

39.

Martin, T.E., Boyce, A.J., Fierro-Calderon, K., Mitchell, A.E., Armstad, C.E., Mouton, J.C. et al. (2017). Enclosed nests may provide greater thermal than nest predation benefits compared with open nests across latitudes. Functional Ecology, 31, 1231-1240.

40.

Matysiokova, B. \& Remeš, V. (2018). Evolution of parental activity at the nest is shaped by the risk of nest predation and ambient temperature across bird species. Evolution , 72, 2214-2224.

41.

Medina, I. (2019). The role of the environment in the evolution of nest shape in Australian passerines. Scientific reports, 9 .

42.

Møller, A.P. (2009). Successful city dwellers: a comparative study of the ecological characteristics of urban birds in the Western Palearctic.Oecologia , 159, 849-858.

43.

Orme, D. (2013). The caper package: comparative analysis of phylogenetics and evolution in R. $R$ package version, 5, 1-36.

44.

Plummer, M., Best, N., Cowles, K. \& Vines, K. (2006). CODA: convergence diagnosis and output analysis for MCMC. $R$ news, 6, 7-11.

45.

Price, J.J. \& Griffith, S.C. (2017). Open cup nests evolved from roofed nests in the early passerines. Proceedings of the Royal Society of London B: Biological Sciences , 284, 20162708.

46.

Purvis, A., Gittleman, J.L., Cowlishaw, G. \& Mace, G.M. (2000). Predicting extinction risk in declining species. Proceedings of the royal society of London. Series B: Biological Sciences , 267, 1947-1952. 
47.

Rabosky, D.L. (2010). Extinction rates should not be estimated from molecular phylogenies. Evolution: International Journal of Organic Evolution, 64, 1816-1824.

48.

Rabosky, D.L. (2017). Phylogenetic tests for evolutionary innovation: the problematic link between key innovations and exceptional diversification. Philosophical Transactions of the Royal Society B: Biological Sciences , 372, 20160417.

49.

Reynolds, S.J., Ibáñez-Álamo, J.D., Sumasgutner, P. \& Mainwaring, M.C. (2019). Urbanisation and nest building in birds: a review of threats and opportunities. Journal of Ornithology, 1-20.

50.

Rosenzweig, M.L. (1995). Species diversity in space and time . Cambridge University Press.

51.

Ross, L., Gardner, A., Hardy, N. \& West, S.A. (2013). Ecology, not the genetics of sex determination, determines who helps in eusocial populations. Current Biology , 23, 2383-2387.

52.

Schliep, K.P. (2011). phangorn: phylogenetic analysis in R.Bioinformatics , 27, 592.

53.

Sheard, C., Neate-Clegg, M.H., Alioravainen, N., Jones, S.E., Vincent, C., MacGregor, H.E. et al. (2020). Ecological drivers of global gradients in avian dispersal inferred from wing morphology. Nature communications , 11, 1-9.

54.

Slagsvold, T. (1989). On the evolution of clutch size and nest size in passerine birds. Oecologia , 79, 300-305. 55.

Sol, D., González-Lagos, C., Moreira, D., Maspons, J. \& Lapiedra, O. (2014). Urbanisation tolerance and the loss of avian diversity.Ecology letters , 17, 942-950.

56.

Stroud, J.T. \& Losos, J.B. (2016). Ecological opportunity and adaptive radiation. Annual Review of Ecology, Evolution, and Systematics , 47.

57.

Wilman, H., Belmaker, J., Simpson, J., de la Rosa, C., Rivadeneira, M.M. \& Jetz, W. (2014). EltonTraits 1.0: Species-level foraging attributes of the world's birds and mammals: Ecological Archives E095-178.Ecology , 95, 2027-2027.

58.

World, B.I.a.H.o.t.B.o.t. (2019). Bird species distribution maps of the world. Version 2019.1. Available at http://datazone.birdlife.org/species/requestdis.

59. 
Yu, G., Smith, D.K., Zhu, H., Guan, Y. \& Lam, T.T.Y. (2017). ggtree: an R package for visualization and annotation of phylogenetic trees with their covariates and other associated data. Methods in Ecology and Evolution , 8, 28-36.

Table 1. Results of PGLS models testing the association between nest type and a) range size (log), b) Temperature niche width (PC1) and c) Precipitation niche width (PC1), for continental species. Estimate, t-value and $\mathrm{P}$-value from model with MCC tree as phylogenetic control. In case where the MCC model showed significant results, we also present the 95\% HPD interval of the estimate across 100 phylogenetic trees.

\begin{tabular}{lllll}
\hline Predictor & Range size (n= 2583 spp.) & Range size (n= 2583 spp.) & Range size (n= 2583 spp.) & Rang \\
\hline & Estimate mcc & t-value & P-value & HPD \\
Open vs Domed & -0.57 & -4.3812 & 0.001 & -0.36 \\
Open vs Cavity & 0.324 & 2.635 & 0.008 & 0.258 \\
log(body size) & -0.225 & -3.304 & $<0.001$ & -0.244 \\
abs(latitude) & 0.046 & 17.180 & $<0.001$ & 0.047 \\
\hline
\end{tabular}

\section{Figures}

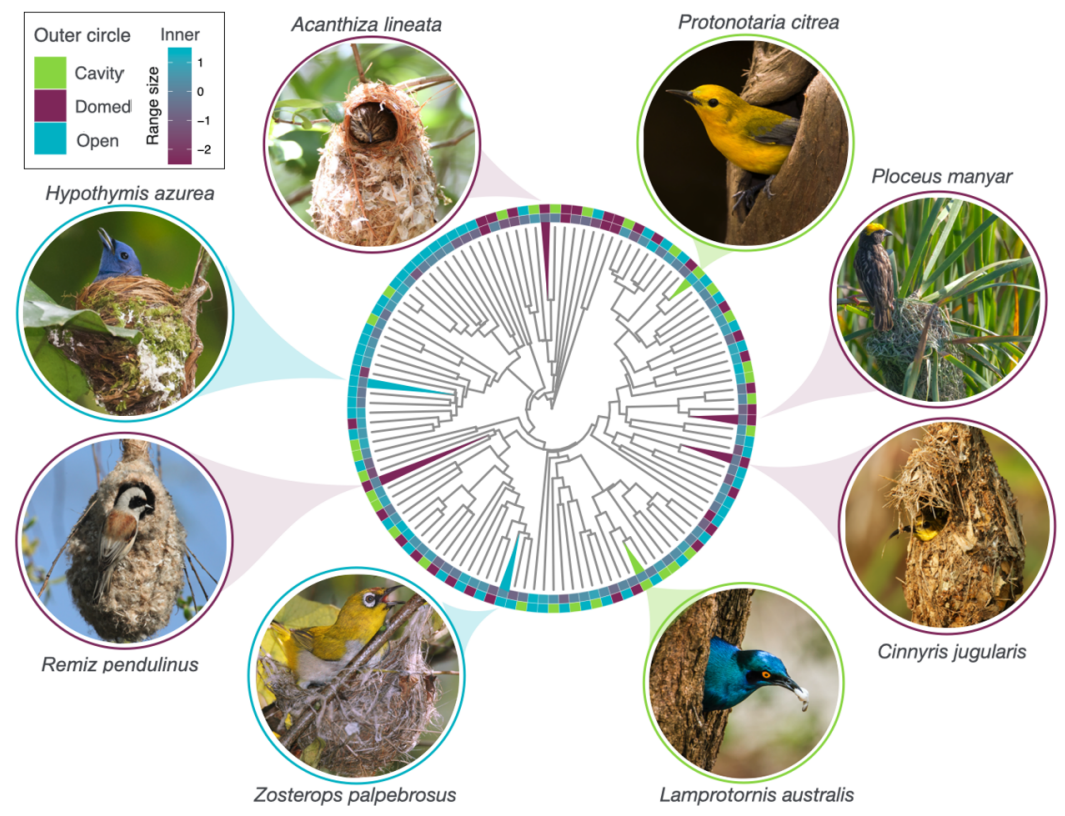

Figure 1. Phylogenetic distribution of nest types and association with range size across 3174 species of songbirds. Tips represent families in most cases, except when there is more than one nest type per family, in which case the tip is divided. Pictures from iStockphoto.com, except for Hypothymis azurea and Ploceus manyar, taken by Damien Esquerré. Figure prepared using

R package ggtree ( $\mathrm{Yu}$ et al. 2017). 


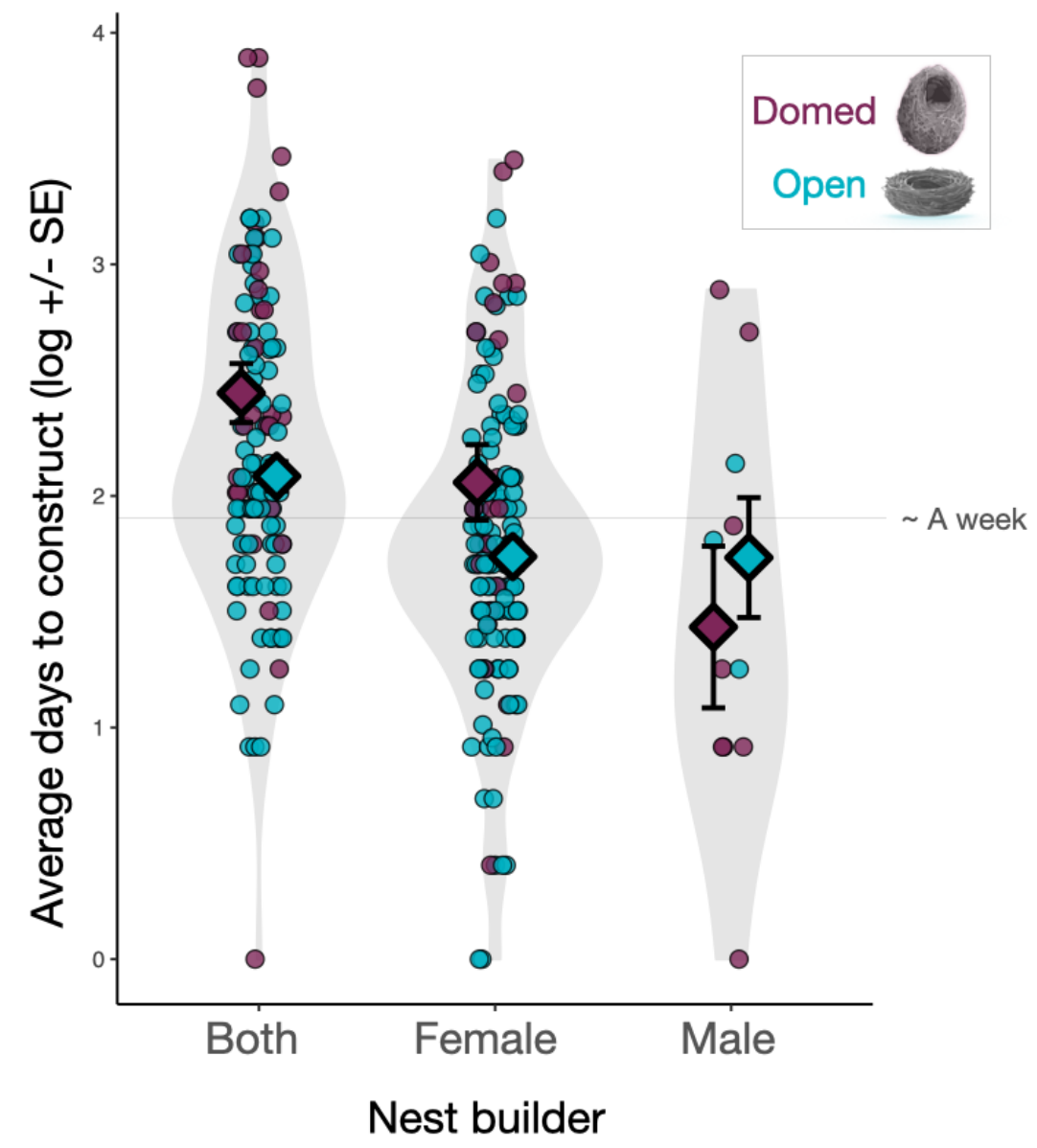

Figure 2. Differences in time spent nest building for songbird species with domed and open nests ( $\mathrm{n}=277)$.
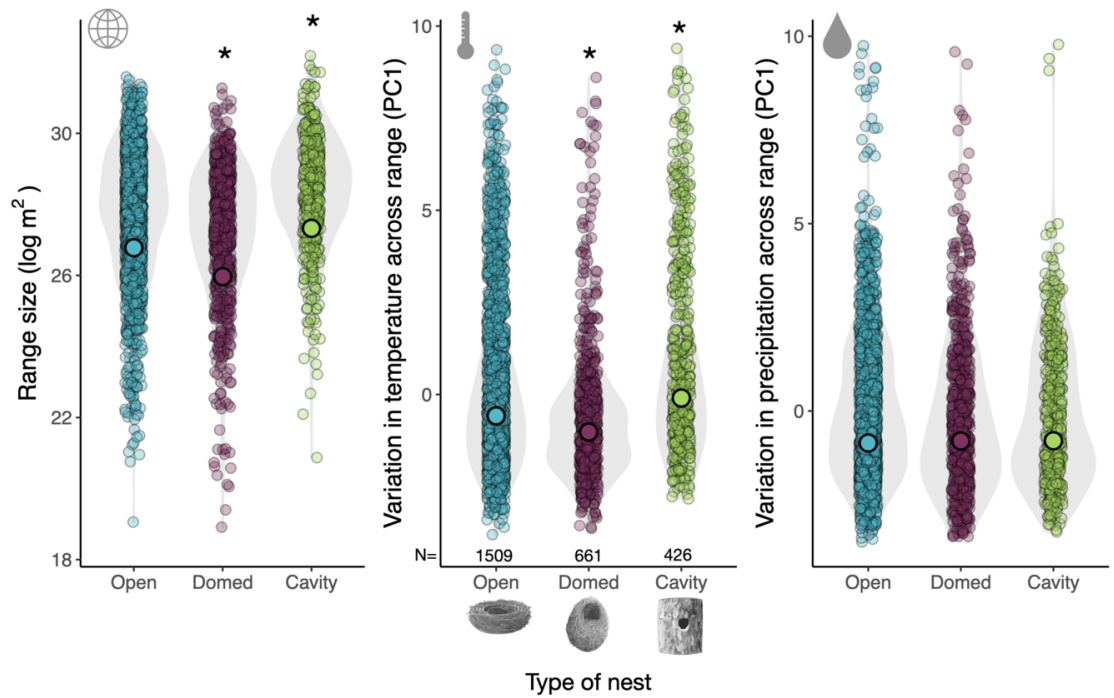
Figure 3. Association between nest type and range size, thermal niche width and precipitation niche width. Smaller transparent points represent raw values per species and larger solid points represent the estimates predicted from the PGLS model in table 1 (for continental species). Illustrations of nests by DP.

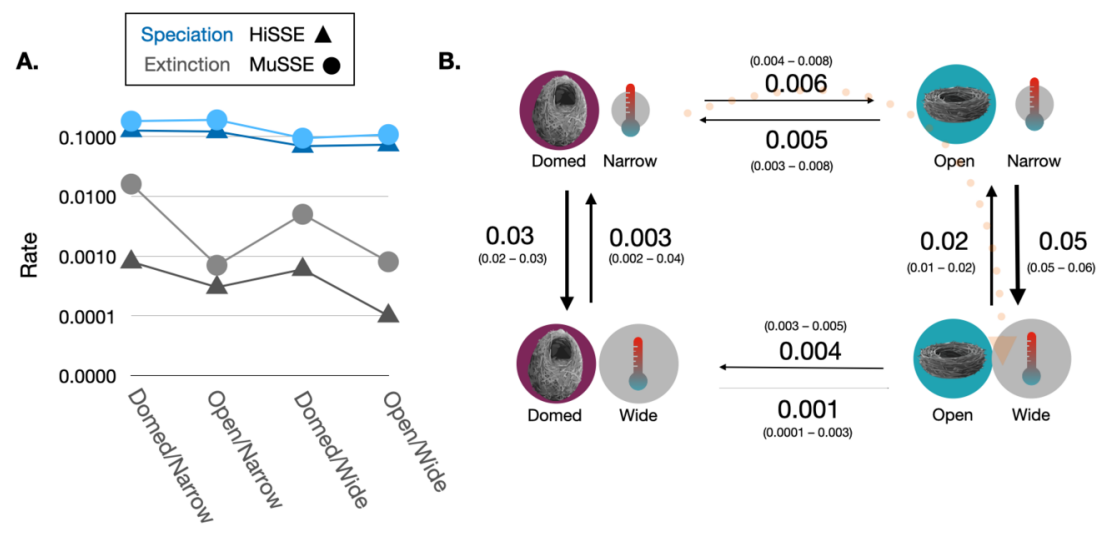

Figure 4. A. Estimates of speciation and extinction rates from HiSSE and MuSSE analyses (RevBayes) for four states of nest type/thermal range combination, using the MCC tree. B. Transition rates between the four states presented in panel A, from the analysis on the MCC tree using RevBayes (HiSSE). Numbers in parentheses represent the $95 \%$ confidence intervals of the rates. Orange arrow in the back represents most likely path, from the most probable ancestral state (domed nest/narrow niche) to the derived and most common condition (open/wide). 